\title{
Underlying mechanism for exchange bias in single-molecule magnetic junctions
}

\author{
Yu-Hui Tang $\oplus^{*}$ and Bao-Huei Huang \\ Department of Physics, National Central University, Jung-Li 32001, Taiwan
}

(Received 1 May 2021; revised 25 August 2021; accepted 9 September 2021; published 20 September 2021)

\begin{abstract}
Magnetic proximity has been observed in a variety of solid-state magnetic devices, but has been less discussed at the molecular scale. In this study, the magnetotransport calculation is carried out using the generalized Landau-Lifshitz-Gilbert (LLG) equation combined with density functional theory (DFT) and our self-developed JUNPY calculated spin-torque effect. Except for the current driven spin torque, which is a promising approach for magnetization switch in magnetic random access memory, the equilibrium fieldlike spin torque also plays a crucial role in the strain-controlled exchange bias with current-controlled magnetic coercivity in single-molecule magnetic junctions. The tight-binding model is further employed to clarify the critical role of the interfacial spin filter effect arising from the hybridization between the linker and Co apex. These multidisciplinary DFT+JUNPY+LLG results may provide important and practical implications in the dual control of magnetic proximity and magnetization switching in molecular spintronics at low temperature, either by tensile strain or via smaller applied current density of the order of $\mathrm{MA} / \mathrm{cm}^{2}$.
\end{abstract}

DOI: 10.1103/PhysRevResearch.3.033264

\section{INTRODUCTION}

When two materials are brought into close proximity, the magnetic proximity effects [1,2], including the exchange anisotropy, exchange bias (EB), and coercivity, are among the most fascinating and active materials research areas today. Among them, the interlayer exchange coupling (IEC) plays a crucial role to manipulate the magnetic hysteresis loop in a variety of solid-state magnetic heterostructures, including the ferromagnetic/antiferromagnetic (FM/AFM) interface [3,4], van der Waals heterostructures [5], $\mathrm{Fe} / \mathrm{MgO}$ interface [6] via interfacial oxidation concentration, and $\mathrm{Pt} / \mathrm{Co} / \mathrm{IrMn}$ trilayer [7] by current-driven spin-orbit torque. Such IEC can be understood by the equilibrium fieldlike spin torque (FLST) of magnetic tunnel junctions (MTJs) in noncollinear magnetic configuration $[8,9]$ and has been predicted to be strongly enhanced in the spin-filter (SF)-based magnetic tunnel junctions [10-12] via the spin-polarized multireflection process.

The other impact of magnetic proximity is the spin polarization at the $\mathrm{FM} /$ barrier interfaces in magnetic tunnel junctions, which not only determines the spin-polarized tunneling current but also raises intensive interest in the so-called inverse magnetoresistance (IMR) effect in $\mathrm{LSMO} / \mathrm{SrTiO}_{3} / \mathrm{Co}$ [13] and LSMO/ $\mathrm{Alq}_{3} / \mathrm{Co}$ [14] asymmetric MTJs. Based on previous theoretical works [15-17], the current-driven spin transfer torque (STT) of noncollinear MTJs can be understood by the interplay of spin-polarized current density between parallel and antiparallel magnetic configurations. Such inter-

\footnotetext{
*yhtang@cc.ncu.edu.tw

Published by the American Physical Society under the terms of the Creative Commons Attribution 4.0 International license. Further distribution of this work must maintain attribution to the author(s) and the published article's title, journal citation, and DOI.
}

facial spin polarization at the FM/barrier is also determinative for the current-driven STT effect in noncollinear magnetic heterostructures.

Recently, intensive research has been transferred from the oxide-based MTJs to the molecular spintronics invoking the role of molecular layers in tuning the interfacial spin-filter (ISF) effect, known as spinterface [18-21]. The weaker spin relaxation mechanism within the central molecule, which allows the electron spin state to be preserved with larger spin diffusion lengths of the order of hundreds of nanometers, along with the diversity and flexibility of molecular synthesis provide the multifunctionality of organic-based magnetic junctions. Most experimental [22-26] and theoretical [27-30] studies focus on the magnetoresistance and the spin-polarized transport in collinear magnetic configurations, but only a few theoretical works report the noncollinear spin-torque effect $[31,32]$. How to characterize the magnetic proximity effects at the molecular scale and modulate the magnetization switching in real organic spintronics devices via the spin-torque effect remains an open question.

Much effort has been focused on the single-molecule building to minimize the size of nanoscale electronics due to its diversity and flexibility. The breaking junction techniques [33-36], invoking the scanning tunneling microscope breaking junction (STM-BJ) and the mechanically controllable breaking junction (MCBJ) processes, have successfully fabricated the single-molecule junctions and have been further applied to investigate the mechanical control over conductance and magnetoresistance in single-molecule magnetic junctions (SMMJs). In this article, we propose two kinds of SMMJ with dissociated amine-ended (BDA) and thiol-ended (BDT) 1,4-Benzenediamine to investigate the multicontrol of magnetization switching via mechanical strain, current densities, and magnetic field. Particularly, we try to answer several questions: How does the linker affect the magnetization switching at the molecular scale? How does mechanical 


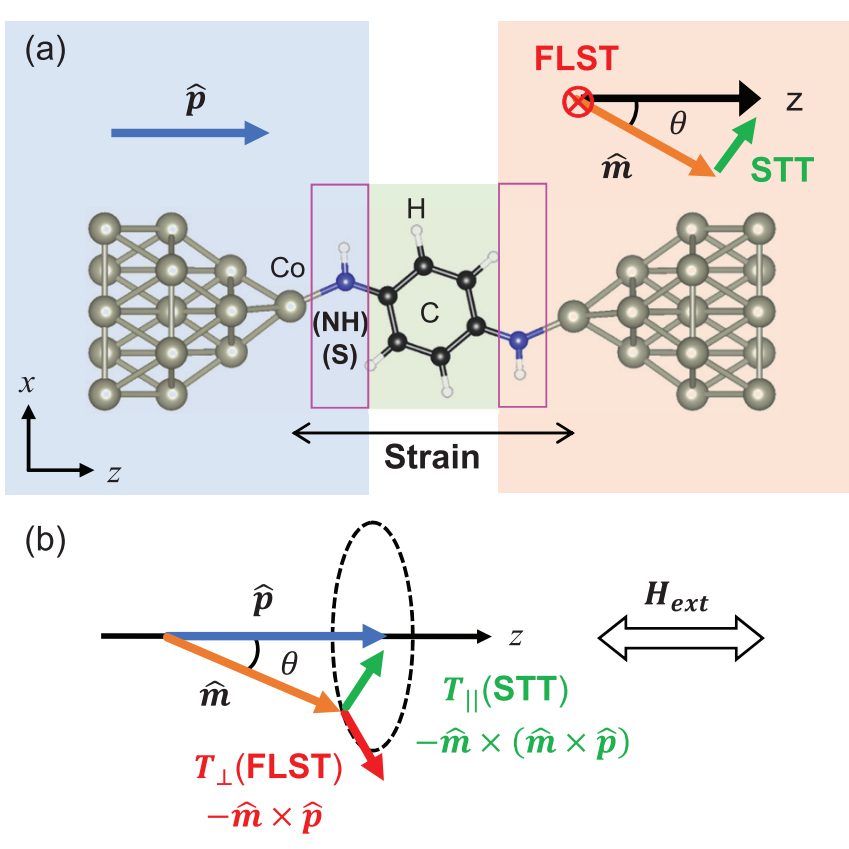

FIG. 1. (a) Junction geometry of the dissociated $\mathrm{Co} / \mathrm{BDA}(\mathrm{BDT}) / \mathrm{Co} \mathrm{SMMJ}$ with $\mathrm{NH}$ (S) linker is expressed in a two-probe device. $\hat{\mathbf{p}}$ and $\hat{\mathbf{m}}$ are the unit vectors of magnetization of the left (fixed) and right (free) Co nanowires and linkers, respectively. $\hat{\mathbf{p}}$ is fixed along the $z$ direction, while $\hat{\mathbf{m}}$ is freely rotated by an angle $\theta$ around the $y$ axis with respect to the $z$ axis to form a noncollinear magnetic configuration. (b) Schematic of macrospin assumption with in-plane external magnetic field, $\mathbf{H}_{\text {ext }}$, and net damplike $\mathbf{T}_{\|}$(STT) and fieldlike $\mathbf{T}_{\perp}$ (FLST) components of spin torque acting on the magnetization of the right $\mathrm{Co}$.

strain affect the magnitude as well as the sign of threshold magnetic fields required for magnetization switching? What are the reasons behind obtaining a strong EB effect with strain-controlled magnetic coercivity in the BDA case and strain-assisted sign reversal of EB with current-controlled magnetic coercivity in the BDT case? The magnetotransport calculations are carried out using the generalized LandauLifshitz-Gilbert (LLG) equation including the equilibrium FLST field and current-driven spin-torque fields, which can be directly obtained by the self-consistent Hamiltonian of the first-principles calculation and the spin transport theory of our self-developed JUNPY package $[32,37,38]$.

\section{METHODOLOGY}

We display, in Fig. 1(a), a prototype of $\mathrm{Co} / \mathrm{BDA}(\mathrm{BDT}) / \mathrm{Co}$ SMMJ, where the dissociated 1,4-Benzenediamine with $\mathrm{NH}$ (S) linker is sandwiched between two semi-infinite hcp [0001] orientated Co tiplike nanowires. To form a noncollinear magnetic configuration, the magnetization direction of the left $\mathrm{Co}$ nanowire, $\hat{p}$, is fixed along the $z$ direction, and the right, $\hat{m}$, is freely rotated by an angle $\theta$ with respect to the $z$ axis. The optimized junction geometries are carried out by the QUANTUM ESPRESSO package [39] based on the density functional theory (DFT) with the generalized gradient approximation (GGA) of Perdew-Burke-Ernzerhof (PBE) exchange-correlation functional [40], the cutoff energy of the plane wave basis of $40 \mathrm{Ry}$,
$\Gamma$ point sampling, and the force criteria of $10^{-3} \mathrm{Ry} / a_{0}$, where $a_{0}$ denotes the Bohr radius. To simulate the stretching process via the breaking junction technique, we apply a tensile strain $\epsilon$ along the $z$ direction to increase the distance between the left and right Co tip atoms from its equilibrium value of $8.7 \AA$ (9.1 $\AA$ ) for Co/BDA(BDT)/Co SMMJ, and then we redo the structural optimization for all strained cases.

Our self-developed JUNPY package [32,37,38] combined with the self-consistent device Hamiltonian and the nonequilibrium Green's function method is first employed to obtain the angle and current dependence of net STT and FLST, i.e., $\mathbf{T}_{\|}(\theta, I)$ and $\mathbf{T}_{\perp}(\theta, I)$, acting on the free layer magnetization, as presented in Fig. 1. Here, $T_{\|}$and $T_{\perp}$ are defined in the directions of $-\hat{\mathbf{m}} \times(\hat{\mathbf{m}} \times \hat{\mathbf{p}})$ and $-\hat{\mathbf{m}} \times \hat{\mathbf{p}}$, respectively. The self-consistent device Hamiltonian of a two-probe system in the noncollinear magnetic configuration with various values of angle is calculated by the DFT-based NANODCAL transport package [41-43], where the double- $\zeta$ double-polarized basis sets of the linear combination of atomic orbitals (LCAO) basis with local density approximation exchange-correlation functional and the $\Gamma$ point sampling are applied.

The charge current and spin current between two atomic sites $i$ and $j$ [37] can be calculated by

$$
I_{i, j}=-\frac{e}{h} \int \operatorname{Tr}\left[\hat{\mathscr{H}}_{i, j} \hat{G}_{j, i}^{<}-\hat{G}_{i, j}^{<} \hat{\mathscr{H}}_{j, i}\right] d E
$$

and

$$
\mathbf{Q}_{i, j}=\frac{1}{4 \pi} \int \operatorname{Tr}\left[\hat{\mathscr{H}}_{i, j} \hat{G}_{j, i}^{<}-\hat{G}_{i, j}^{<} \hat{\mathscr{H}}_{j, i}\right] \sigma d E,
$$

in which the directions of the current and electron flow are along $+z$ and $-z$, respectively, under a positive bias. Here, $\hat{\mathscr{H}} \equiv(\hat{H}-E \hat{S})$ is the reduced Hamiltonian, $\hat{H}$ is the device Hamiltonian, $\hat{S}$ is the overlapping integral, $\hat{G}^{<}$is the Keldysh Green's function, $\sigma=\left(\sigma_{x}, \sigma_{y}, \sigma_{z}\right)$ is the vector of Pauli matrices, and the energy integral is over occupied states and nonequilibrium bias window. We next define $\mathbf{Q}_{n}$ as the spin current passing through an interface $n$, namely, $\mathbf{Q}_{n}=$ $\sum_{i<n} \sum_{j>n} \mathbf{Q}_{i, j}$. The net FLST and STT acting on the right (free) Co electrode can be simply obtained by $T_{\perp}=Q_{0}^{y}$ and $T_{\|}=-Q_{0}^{x} \cos \theta+Q_{0}^{z} \sin \theta$, where $n=0$ denotes the interface between the right $\mathrm{N} / \mathrm{S}$ ion and the right $\mathrm{Co}$ adatom.

The generalized Landau-Lifshitz-Gilbert (LLG) equation with both the STT and FLST components of noncollinear spin-torque effects [44] can be expressed by

$$
\begin{aligned}
\frac{d \hat{\mathbf{m}}}{d t}= & -\gamma\left(\hat{\mathbf{m}} \times \mathbf{H}_{\text {eff }}\right)+\alpha\left(\hat{\mathbf{m}} \times \frac{d \hat{\mathbf{m}}}{d t}\right) \\
& -\gamma \hat{\mathbf{m}} \times\left(\hat{\mathbf{m}} \times H_{\|} \hat{\mathbf{p}}\right)-\gamma\left(\hat{\mathbf{m}} \times H_{\perp} \hat{\mathbf{p}}\right)
\end{aligned}
$$

or, equivalently,

$$
\begin{aligned}
\frac{1+\alpha^{2}}{\gamma} \frac{d \hat{\mathbf{m}}}{d t}= & -\hat{\mathbf{m}} \times\left(\mathbf{H}_{\text {eff }}+\mathbf{H}_{\text {pre }}\right) \\
& -\hat{\mathbf{m}} \times\left[\hat{\mathbf{m}} \times \alpha\left(\mathbf{H}_{\text {eff }}+\mathbf{H}_{\text {damp }}\right)\right],
\end{aligned}
$$

where $\mathbf{H}_{\text {pre }}=\left(-\alpha H_{\|}+H_{\perp}\right) \hat{\mathbf{p}}, \mathbf{H}_{\mathbf{d a m p}}=\left(H_{\|} / \alpha+H_{\perp}\right) \hat{\mathbf{p}}, \gamma$ is the gyromagnetic ratio, and $\alpha$ is the Gilbert damping constant. Note that $\mathbf{H}_{\text {damp }}$ and $\mathbf{H}_{\text {pre }}$ are introduced to represent the effective fields along the damping and precession directions, respectively, for the right Co electrode. The magnetic 
(a)

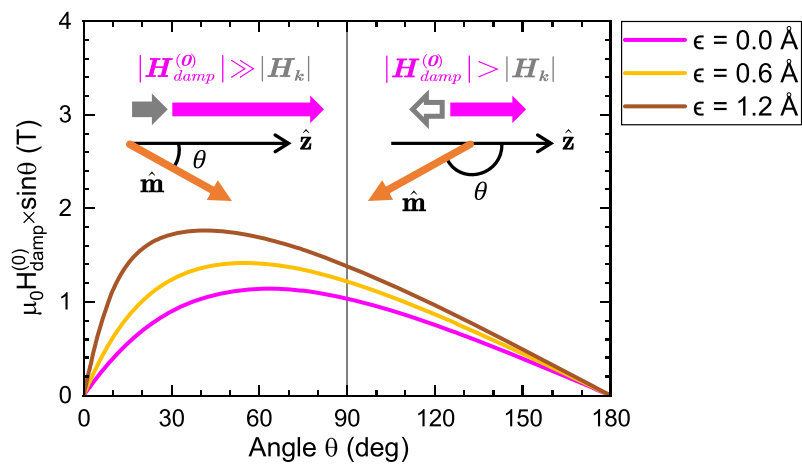

(c)

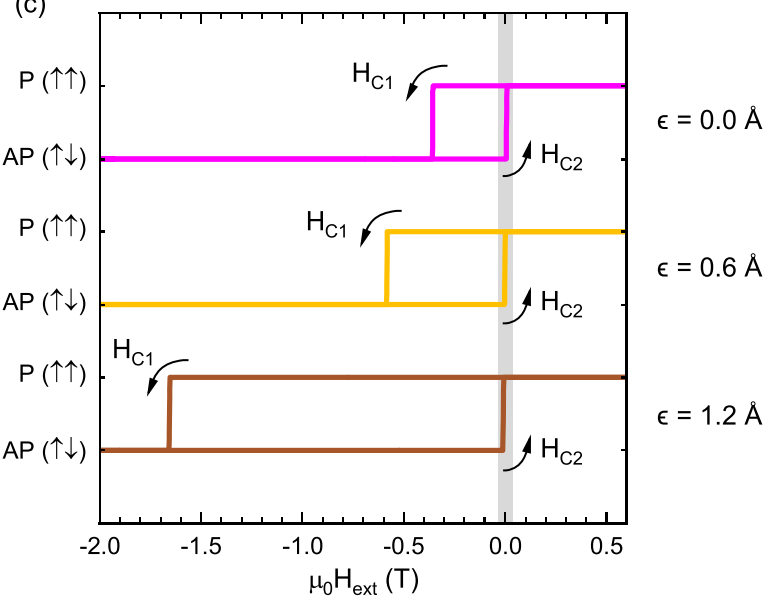

(b)

Co/BDT/Co
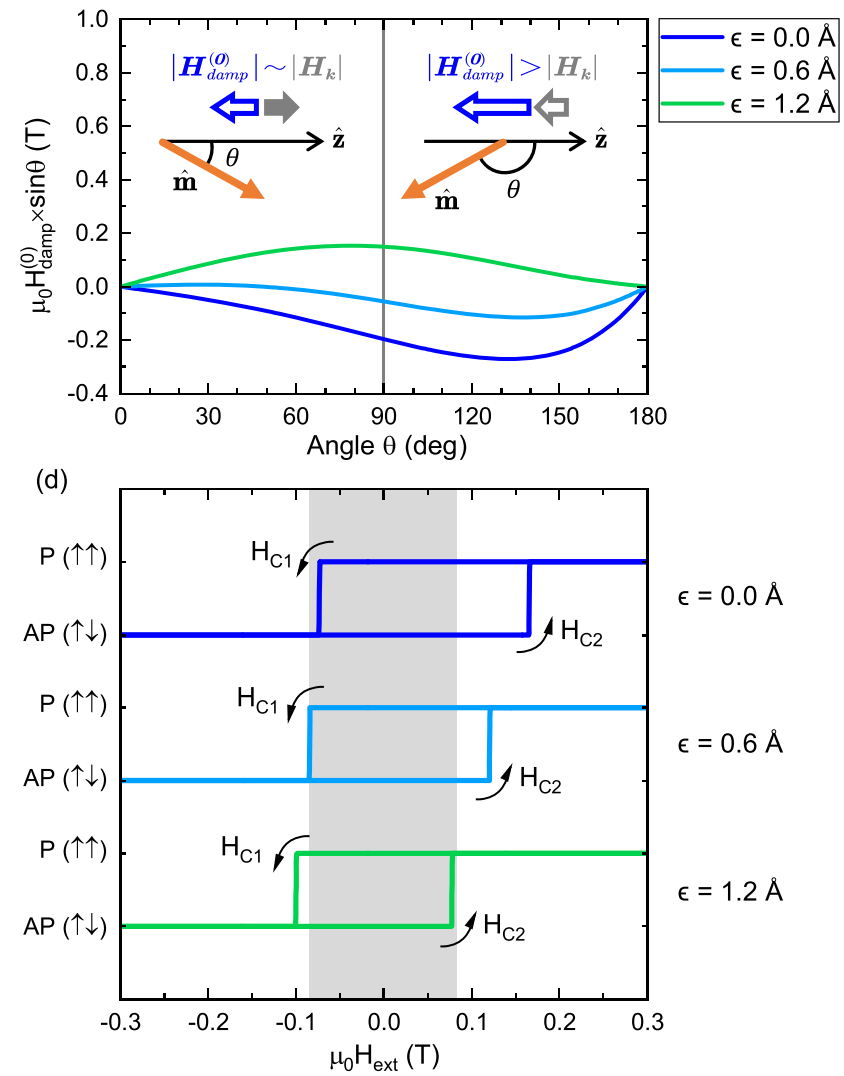

FIG. 2. Angle dependence of the DFT+JUNPY calculated equilibrium FLST field, $H_{\text {damp }}^{(0)} \sin \theta$, without an applied current for (a) $\mathrm{Co} / \mathrm{BDA} / \mathrm{Co}$ and (b) Co/BDT/Co SMMJs under various tensile strains ( $\epsilon$ ). The insets are the schematic plots of $H_{\text {damp }}^{(0)}$ and $H_{k}$ in unstrained (a) BDA and BDT cases when $\theta$ is below and above $90^{\circ}$, where $H_{k}$ is the cohesive field of the right Co electrode. The DFT+JUNPY+LLG calculated magnetization hysteresis curves $\left(m_{z}-H\right)$ for (c) Co/BDA/Co and (d) Co/BDT/Co SMMJs under various tensile strains at zero temperature. The gray shaded area represents the field region between $\pm \mu_{0} H_{k}$. The $H_{\mathrm{C} 1}\left(H_{\mathrm{C} 2}\right)$ is the threshold field required for P-to-AP (AP-to-P) magnetization switching, where $\mathrm{P}$ and AP denote the parallel $\left(\theta=0^{\circ}\right)$ and antiparallel $\left(\theta=180^{\circ}\right)$, respectively.

potential energy of the right Co electrode is given as $U=$ $U_{K}+U_{H}$, where $U_{K}=(1 / 2) \mu_{0} M_{s} H_{k} \sin ^{2} \theta$ is from the uniaxial anisotropy, $U_{H}=-\mathbf{M} \cdot \mathbf{H}_{\mathrm{ext}}$ is from an external magnetic field, and $\mathbf{M}=M_{S} \hat{\mathbf{m}}$. Given the potential energy $U$, the effective field $\mathbf{H}_{\mathrm{eff}}$ is expressed as $\mathbf{H}_{\mathrm{eff}}=-\partial U / \partial \mathbf{M}$. Moreover, the effective fields induced by both STT and FLST are $H_{\|, \perp} \sin \theta=T_{\|, \perp} /\left(\mu_{0} M_{s} t_{F} A\right)$, where $M_{s}, t_{F}$, and $A$ are the volume magnetization saturation parameter, the thickness, and the lateral area of the right Co electrode. For the Co electrode [45], we choose $\alpha=0.01, \mu_{0} H_{k}=76 \mathrm{mT}, M_{s}=1.27 \times 10^{6}$ $\mathrm{A} / \mathrm{m}$, the thickness $t_{F}=100 \mathrm{~nm}$, and the lateral area $A=$ $1.63 \times 10^{-19} \mathrm{~m}^{2}$. Note here that LLG simulations are carried out by the Runge-Kutta fourth-order method with a fixed time step of $10 \mathrm{ps}$ and a cumulative integration time of $0.1 \mu \mathrm{s}$ for each field point.

\section{RESULTS AND DISCUSSION}

\section{A. EB effect with equilibrium FLST field: DFT+JUNPY+LLG}

We first discuss the effect of the equilibrium FLST field on magnetization switching in SMMJs. Recalling the spin-torque effect in conventional MTJs [8,9] without applying current, only the equilibrium FLST exists but the current driven STT vanishes, and thus the $\mathbf{H}_{\text {pre }}$ and $\mathbf{H}_{\text {damp }}$ in Eq. (4) are solely determined by the equilibrium FLST field $H_{\perp}^{(0)}$, i.e., $\mathbf{H}_{\text {pre }}^{(0)}=$ $\mathbf{H}_{\text {damp }}^{(0)}=H_{\perp}^{(0)} \hat{\mathbf{p}}$. Notice that the positive and negative magnitudes of $H_{\perp}^{(0)}$ denote the fieldlike and antifieldlike equilibrium fields, respectively. In Figs. 2(a) and 2(b), we present the angle dependence of $H_{\mathrm{damp}}^{(0)} \sin \theta$ for $\mathrm{Co} / \mathrm{BDA} / \mathrm{Co}$ and $\mathrm{Co} / \mathrm{BDT} / \mathrm{Co}$ SMMJs under various tensile strains at zero temperature, respectively, where the central BDA (BDT) molecule is connected by the $\mathrm{NH}(\mathrm{S})$ linker and the Co electrode. It is intriguing to find that equilibrium FLST fields of the BDA cases exhibit nonsinusoidal angular dependence with positive maxima below $90^{\circ}$, which are about two orders larger than the cohesive field $H_{k}$ of the Co electrode and can be enlarged by a tensile strain. When the linker is replaced by the $S$ ion in BDT cases, surprisingly, we can find a strain-controlled nonsinusoidal angular dependence of the equilibrium FLST field, which varies from a negative minimum above $90^{\circ}$ without strain $(\epsilon=0)$ to a positive maximum around $90^{\circ}$ under a tensile strain of $\epsilon=1.2 \AA$.

To clarify how the equilibrium FLST field influences the magnetization switching by choosing the linker and applying tensile strain, we present, in Figs. 2(c) and 2(d), the DFT + JUNPY + LLG calculated magnetization hysteresis curve $\left(m_{z}-\mu_{0} H_{\mathrm{ext}}\right)$ for $\mathrm{Co} / \mathrm{BDA} / \mathrm{Co}$ and $\mathrm{Co} / \mathrm{BDT} / \mathrm{Co} \mathrm{SMMJ}$ under 
various tensile strains, respectively. The gray shaded area represents the field region between $\pm \mu_{0} H_{k}$, and $H_{\mathrm{C} 1}\left(H_{\mathrm{C} 2}\right)$ is the threshold field required for P-to-AP (AP-to-P) magnetization switching, where $\mathrm{P}$ and AP refer to the parallel $\left(\theta=0^{\circ}\right)$ and antiparallel $\left(\theta=180^{\circ}\right)$ magnetic configurations, respectively. For the BDA cases presented in Fig. 2(a), the large positive value of $H_{\text {damp }}^{(0)}$ with a maximum below $90^{\circ}$ strongly supports (resists) the cohesive field $H_{k}$ of the Co electrode below (above) $90^{\circ}$, and hence in turn determines the precession angle $\theta$ and magnetization switching threshold criterion. As shown in Fig. 2(c), such strong and positive $H_{\text {damp }}^{(0)}$ not only significantly delays the P-to-AP, but also assists the AP-to-P magnetization switching, and hence in turn moves the threshold field $H_{\mathrm{C} 1}\left(H_{\mathrm{C} 2}\right)$ for P-to-AP (AP-to-P) toward a more (less) negative field. Moreover, when a tensile strain is applied, the maximum of positive $H_{\text {damp }}^{(0)}$ can be further enlarged and shifted to smaller angle $\theta$. This thus results in the significant $\mathrm{EB}$ along with the strain-enhanced magnetic coercivity in $\mathrm{Co} / \mathrm{BDA} / \mathrm{Co}$ SMMJs.

In contrast, for the unstrained BDT $(\epsilon=0)$ case displayed in Fig. 2(b), the negative value of $H_{\text {damp }}^{(0)}$ with minimum above $90^{\circ}$, which is about one order larger than $H_{k}$, becomes against (supporting) the cohesive field $H_{k}$ of the Co electrode below (above) $90^{\circ}$. As shown in Fig. 2(d), this slightly assists the P-to-AP but strongly delays the AP-to-P magnetization switching, and thus causes the shift of $H_{\mathrm{C} 1}\left(H_{\mathrm{C} 2}\right)$ toward a less (more) positive field. More interestingly, applying a tensile strain can even promote a sign reversal of $H_{\text {damp }}^{(0)}$, i.e., changing from the negative minimum above $90^{\circ}$ in the $\epsilon=0.0 \AA$ case to the positive maximum around $90^{\circ}$ in the $\epsilon=1.2 \AA$ case, and again gives rise to the movement of $H_{\mathrm{C} 1}\left(H_{\mathrm{C} 2}\right)$ toward a less (more) negative field. Therefore, the strain-controlled equilibrium FLST field in Co/BDT/Co SMMJs provides a pathway to modulate the magnetic proximity at the molecular level, including the sign reversal of EB and tunable magnetic coercivity.

\section{B. Role of linker and strain in equilibrium FLST field: TB+JUNPY}

It is natural to raise another question: What is the role of the linker and strain on the angle dependence of the equilibrium FLST field? To answer this, we further propose the onedimensional (1D) single-band tight-binding (TB) model in the $\mathrm{L} / \mathrm{IL} / \operatorname{Ben} / \operatorname{IR}(\theta) / \mathrm{R}(\theta)$ noncollinear magnetic heterostructure [12]. The device Hamiltonian in the noncollinear magnetic configuration is defined as

$$
\hat{H}=\sum_{i j, \sigma} t_{i j, \sigma} \mathbf{c}_{i, \sigma}^{\dagger} \mathbf{c}_{j, \sigma}+\sum_{i, \sigma \sigma^{\prime}} \Delta_{i} \mathbf{c}_{i, \sigma^{\prime}}^{\dagger}\left(\boldsymbol{\sigma} \cdot \hat{\mathbf{M}}_{i}\right) \mathbf{c}_{i, \sigma},
$$

where $\boldsymbol{\sigma}=\left(\sigma_{x}, \sigma_{y}, \sigma_{z}\right)$ is the vector of Pauli matrices and $\hat{\mathbf{M}}_{i}$ represents the magnetization direction on the $i$ site, or, equivalently,

$$
\hat{H}=\hat{H}_{\mathrm{L}}+\hat{H}_{\mathrm{IL}}+\hat{H}_{\mathrm{Ben}}+\hat{H}_{\mathrm{IR}}+\hat{H}_{\mathrm{R}}+\hat{H}_{\mathrm{cpl}} .
$$

The subscripts $\mathrm{L}(\mathrm{R})$ denotes the isolated left (right) Co nanowire, IL (IR) refers to the left (right) $\mathrm{NH}$ or S linker, Ben means central phenyl ring, and $H_{\mathrm{cpl}}$ involves the couplings between two neighboring regions. Here, $t_{i i, \sigma}=\varepsilon_{i}^{\sigma}$ with (a) $\mathrm{Co} / \mathrm{BDA} / \mathrm{Co}$ (DFT)

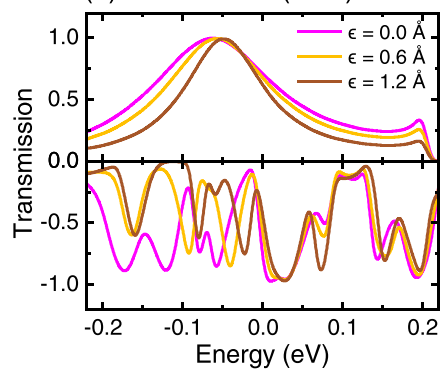

(c) Co/BDA/Co (TB+JunPy)
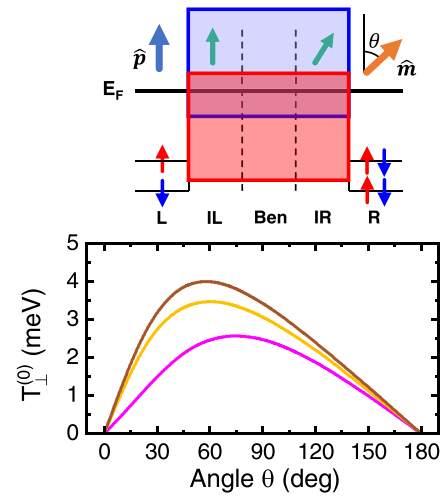

(b) $\mathrm{Co} / \mathrm{BDT} / \mathrm{Co}$ (DFT)

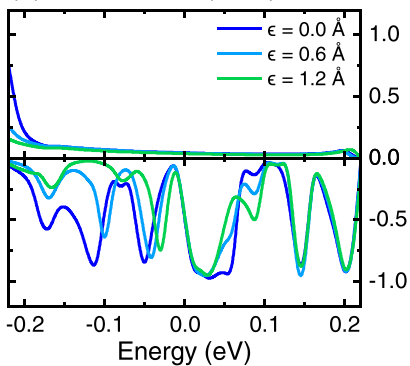

(d) Co/BDT/Co (TB+JunPy)
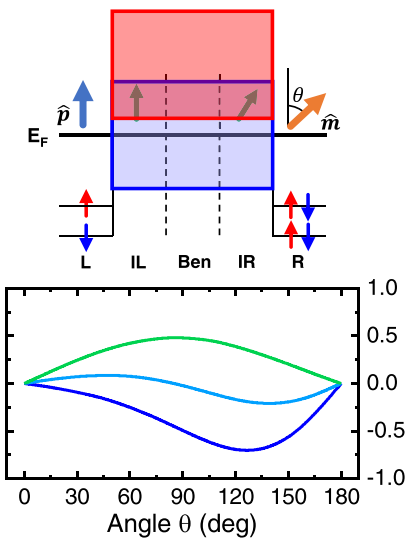

FIG. 3. DFT-calculated spin-polarized transmission spectra (top) for (a) $\mathrm{Co} / \mathrm{BDA} / \mathrm{Co}$ and (b) $\mathrm{Co} / \mathrm{BDT} / \mathrm{Co} \mathrm{SMMJs}$ in parallel $(\theta=0)$ magnetic configuration under various tensile strains $\epsilon$, where $E_{\mathrm{F}}=$ $0.0 \mathrm{eV}$ denotes the Fermi energy. Schematic 1D-TB energy profiles of noncollinear $\mathrm{L} / \mathrm{IL} / \mathrm{Ben} / \operatorname{IR}(\theta) / \mathrm{R}(\theta)$ (middle), and the angle dependence of TB+JUNPY calculated equilibrium FLST spin torque $T_{\perp}^{(0)}$ (bottom), for (c) Co/BDA/Co and (d) Co/BDT/Co SMMJs under various tensile strains. The notations of IL (IR) and Ben refer to the spin-polarized left (right) $\mathrm{NH} / \mathrm{S}$ linker and central phenyl ring, respectively. The red and blue arrows denote the bottom of the spin-up and spin-down energy bands of left (L) and right (R) Co. Since the magnetization $\hat{\mathbf{p}}$ in region $\mathrm{L}$ is fixed in the $+z$ direction, upon rotation of $\hat{\mathbf{m}}$ along $\hat{\mathbf{p}}$, the spin-up (spin-down) energy bands of region $\mathrm{R}$ acquire spin-down (spin-up) energy contributions. The red and the blue shaded areas denote the range of spin-polarized energy levels of the central IL/Ben/IR.

$\sigma=\uparrow, \downarrow$ represents the $\sigma$-spin polarized on-site energy on the $i$ site, the hopping parameter $t=t_{i j, \sigma}$ is nonzero only between nearest-neighboring sites with $j=i \pm 1$, and $\Delta_{i}=\varepsilon_{i}^{\downarrow}-\varepsilon_{i}^{\uparrow}$ denotes the exchange energy on the $i$ site.

We present, in Fig. 3, the DFT-calculated spinpolarized transmission spectra for (a) $\mathrm{Co} / \mathrm{BDA} / \mathrm{Co}$ and (b) $\mathrm{Co} / \mathrm{BDT} / \mathrm{Co}$ SMMJs in parallel $(\theta=0)$ magnetic configuration under various tensile strains. It is clear to find that all three BDA cases exhibit a broad and pronounced spin-up transmission feature near the Fermi energy $\left(E_{\mathrm{F}}=0.0 \mathrm{eV}\right)$, but reducing spiky spin-down transmission peaks with the increase of tensile strain, resulting from the strong (weak) spin-up (spin-down) hard-hard coupling between the Co- $d, \mathrm{~N}-p_{y}$, and $\pi$ orbital of the central phenyl ring to support the spin-polarized electrons injected from the Co electrode into the central molecule via resonant tunneling [46]. However, for the BDT cases, the pronounced spin-up 
TABLE I. The spin-polarized on-site energies used in the $\mathrm{TB}+\mathrm{JUNPY}$ calculation in units of $\mathrm{eV}$ for $\mathrm{Co} / \mathrm{BDA} / \mathrm{Co}$ and $\mathrm{Co} / \mathrm{BDT} / \mathrm{Co} \mathrm{SMMJ}$ under various tensile strains in unit of $\AA$. The nearest-neighbor hopping parameter is $t=-0.2 \mathrm{eV}$ in all regions.

\begin{tabular}{lcccccccc}
\hline \hline & \multicolumn{2}{c}{ Co } & & \multicolumn{2}{c}{ BDA } & & \multicolumn{2}{c}{ BDT } \\
\cline { 2 - 3 } \cline { 7 - 8 } Strain & $\varepsilon_{\mathrm{L}, \mathrm{R}}^{\uparrow}$ & $\varepsilon_{\mathrm{L}, \mathrm{R}}^{\downarrow}$ & & $\varepsilon_{\mathrm{IL}, \mathrm{Ben}, \mathrm{IR}}^{\uparrow}$ & $\varepsilon_{\mathrm{IL}, \mathrm{Ben}, \mathrm{IR}}$ & & $\varepsilon_{\mathrm{IL}, \mathrm{Ben}, \mathrm{IR}}^{\uparrow}$ & $\varepsilon_{\mathrm{IL}, \mathrm{Ben}, \mathrm{IR}}^{\downarrow}$ \\
\hline $0.0 \AA$ & -0.15 & -0.35 & & -0.30 & 0.19 & & 0.55 & 0.00 \\
$0.6 \AA$ & -0.15 & -0.35 & & -0.30 & 0.22 & & 0.55 & 0.03 \\
$1.2 \AA$ & -0.15 & -0.35 & & -0.30 & 0.25 & & 0.55 & 0.06 \\
\hline \hline
\end{tabular}

transmission feature is moved away from the $E_{\mathrm{F}}$ and gives almost no contribution around the $E_{\mathrm{F}}$. Instead, the spiky spin-down transmission peaks near the $E_{\mathrm{F}}$ play a major role in the ISF effect and dominate the resonant tunneling.

To simulate such strong spin-up (weak spin-down) dominated resonant tunneling in $\mathrm{Co} / \mathrm{BDA} / \mathrm{Co}(\mathrm{Co} / \mathrm{BDT} / \mathrm{Co})$ SMMJs, the spin-polarized on-site energies used in the TB+JUNPY calculation are listed in Table I and the nearestneighbor hopping parameter is $t=-0.2 \mathrm{eV}$ in all regions. For BDA cases, we fix the negative $\varepsilon_{\mathrm{IL}, \mathrm{Ben}, \mathrm{IR}}^{\uparrow}$ to represent the broad spin-up feature in all three cases, but we gradually increase the positive $\varepsilon_{\mathrm{IL}, \mathrm{Ben}, \mathrm{IR}}^{\downarrow}$ to simulate the suppression of spin-down transmission features near the $E_{\mathrm{F}}$ with the increase of strain. As for the BDT cases, the positive $\varepsilon_{\mathrm{IL}, \mathrm{Ben}, \mathrm{IR}}^{\uparrow}$ is fixed to modulate the absence of the spin-up resonant channel near the $E_{\mathrm{F}}$, while the much smaller $\varepsilon_{\mathrm{IL}, \mathrm{Ben}, \mathrm{IR}}^{\downarrow}$ represents the weak spin-down resonant tunneling and gradually increases to simulate the suppression of the spin-down transmission feature near the $E_{\mathrm{F}}$ with the application of tensile strain.

The qualitative agreement between the DFT+JUNPY calculation in Figs. 2(a) and 2(b) and the TB+JUNPY simulation in Figs. 3(c) and 3(d) confirms a crucial fact: The origin of the strain effect on the magnitude, sign, and angle dependence of the equilibrium FLST field lies in the spin-up (spin-down) resonance states for BDA (BDT) cases, i.e., the lower red (blue) shaded area within the central IL/Ben/IR region demonstrated in the schematic energy diagrams of Fig. 3. The hard-hard coupling between the N- $p$ (S- $p$ ), Co- $d$, and $\pi$ band of central benzene renders the NH (S) linker as a major role of the ISF layer to dramatically enlarge the IEC as well as the equilibrium FLST field, and thus in turn gives rise to the significant $\mathrm{EB}$ with strain-controlled magnetic coercivity in $\mathrm{Co} / \mathrm{BDA} / \mathrm{Co} \mathrm{SMMJ}$ and the strain-assisted sign reversal of EB with current-controlled magnetic coercivity in $\mathrm{Co} / \mathrm{BDT} / \mathrm{Co}$ SMMJs. This is in sharp contrast to the conventional MTJs, where the insulating barrier well separates two ferromagnetic electrodes and in turn gives a very weak equilibrium FLST field that is usually ignored for magnetization switching.

\section{Current-driven magnetization switching: DFT+JUNPY+LLG}

Next, we turn our discuss to the current-driven magnetization switching in the presence of the linker-assisted ISF effect. Because the $H_{\text {damp }}$ in Eq. (4) is decisive for magnetization switching, here we define $H_{\text {damp }}^{(J)}=H_{\text {damp }}-H_{\perp}^{(0)}=$
$H_{\|} / \alpha+H_{\perp}^{(J)}$ to represent the current-driven spin-torque fields, including both purely current-contributed STT, $H_{\|}$, and current-induced FLST, $H_{\perp}^{(J)}$. We first present, in Fig. 4(a), the angle dependence of DFT+JUNPY calculated $H_{\text {damp }}^{(J)} \sin \theta$ for Co/BDA/Co SMMJs under various strains with an applied current density of $J= \pm 1.8 \mathrm{MA} / \mathrm{cm}^{2}$. In comparison with the above-discussed $\mathrm{NH}$-linker induced strong equilibrium FLST field, i.e., $\mu_{0} H_{\text {damp }}^{(0)}=\mu_{0} H_{\perp}^{(0)} \sim 2$ T, shown in Fig. 2(a), the much smaller current-driven $\mu_{0} H_{\text {damp }}^{(J)} \sim-0.04 \mathrm{~T}$ plays almost no role on the magnetization switching. This is because the equilibrium FLST torque originates from the IEC and can be calculated by the integration over whole occupied states, which are dominated by the broad spin-up transmission spectra shown in Fig. 3(a), but the current-driven $H_{\text {damp }}^{(J)}$ is obtained by the integration solely within the small bias window of the order of $10^{-3} \mathrm{eV}$. We redo the DFT+JUNPY+LLG simulation and display, in Fig. 4(c), the current dependence of the threshold fields, $H_{\mathrm{C} 1, \mathrm{C} 2}(J)$. The nearly current independence of $H_{\mathrm{C} 1, \mathrm{C} 2}$ reveals that the significant $\mathrm{EB}$ effect in $\mathrm{Co} / \mathrm{BDA} / \mathrm{Co}$ SMMJs is purely dominated by the equilibrium FLST field, and the magnetic coercivity can only be controlled by tensile strain but not via applied current density of the order of $\mathrm{MA} / \mathrm{cm}^{2}$.

On the other hand, the angle dependence of DFT+JUNPY calculated $H_{\text {damp }}^{(J)} \sin \theta$ for Co/BDT/Co SMMJs under various strains with applied current densities of $J= \pm 1.8 \mathrm{MA} / \mathrm{cm}^{2}$ are displayed in Fig. 4(b). Clearly, magnitudes of the currentdriven $H_{\text {damp }}^{(J)}$ and the equilibrium FLST field $H_{\text {damp }}^{(0)}$ shown in Fig. 2(b) are of the same order, and thus the interplay between these two spin-torque fields determines the magnetization switching in BDT cases. By following the previous discussion in magnetization switching via the equilibrium FLST field, the nonsinusoidal angular dependence of negative current-driven $H_{\text {damp }}^{(J<0)}$ with a negative minimum above $90^{\circ}$ resists (supports) the cohesive field, $H_{k}$, of the Co electrode below (above) $90^{\circ}$, which causes the movement of $H_{\mathrm{C} 1}\left(H_{\mathrm{C} 2}\right)$ toward a less (more) positive field, as shown in Fig. 4(d). Similarly, the odd parity of $H_{\text {damp }}^{(J)}$ renders the shift of $H_{\mathrm{C} 1}\left(H_{\mathrm{C} 2}\right)$ toward a less (more) negative field. To conclude, the nearly linear current density dependence of $H_{\mathrm{C} 1, \mathrm{C} 2}$ combined with the previously discussed strain-controlled equilibrium FLST field in Co/BDT/Co SMMJs present an important practical implication in the dual control of the EB effect and magnetic coercivity either by tensile strain or via applied current density of the order of $\mathrm{MA} / \mathrm{cm}^{2}$.

Finally, we extend previous discussions to finite temperatures in consideration of the thermal effective field $\mathbf{H}_{\text {th }}$ $[45,47]$, which is used to simulate the thermal fluctuation, and each Cartesian component is chosen at random from a normal distribution with a chosen variance. Specifically, $\left\langle\mathbf{H}_{\mathrm{th}}(t)\right\rangle=0$ and

$$
\left\langle\mathbf{H}_{\mathrm{th}}(t) \mathbf{H}_{\mathrm{th}}\left(t^{\prime}\right)\right\rangle=\frac{2 \alpha k_{B} T}{\gamma \Omega \mu_{0} M_{s}} \delta_{i j} \delta\left(t-t^{\prime}\right),
$$

where $i, j=x, y, z, k_{B}$ is the Boltzmann constant, and $\Omega$ is the free layer volume. Figure 5 shows the phase diagram of the magnetization hysteresis for DFT+JUNPY+LLG calculated (a) $\mathrm{Co} / \mathrm{BDA} / \mathrm{Co}$ and (b) $\mathrm{Co} / \mathrm{BDT} / \mathrm{Co} \mathrm{SMMJs}$ without strain at various temperatures of $T=0,1,10$, and $100 \mathrm{~K}$, in which 
(a)
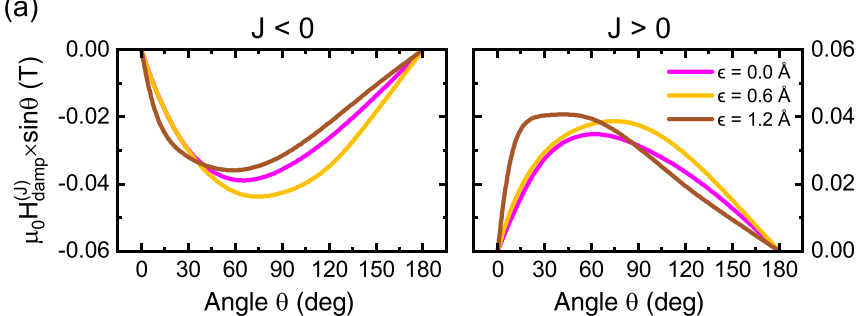

(c)

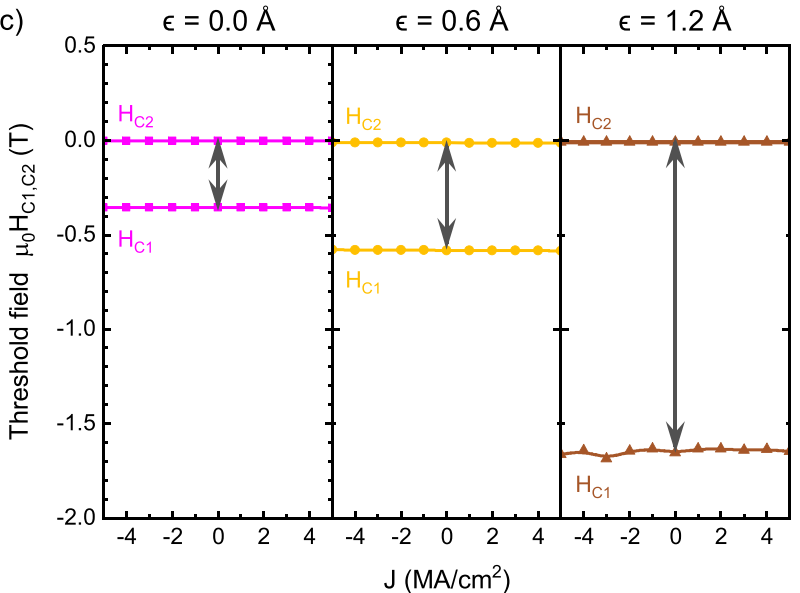

(b)
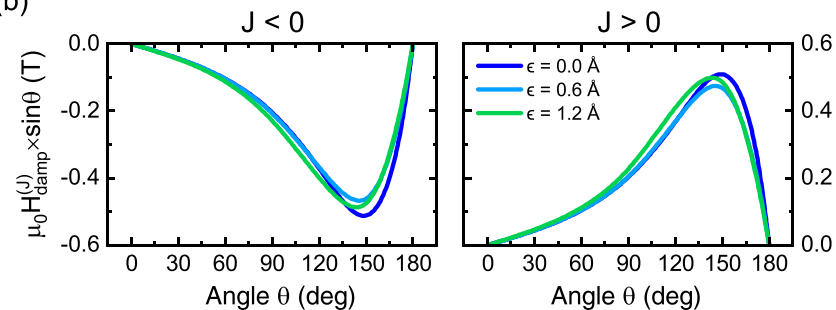

(d)

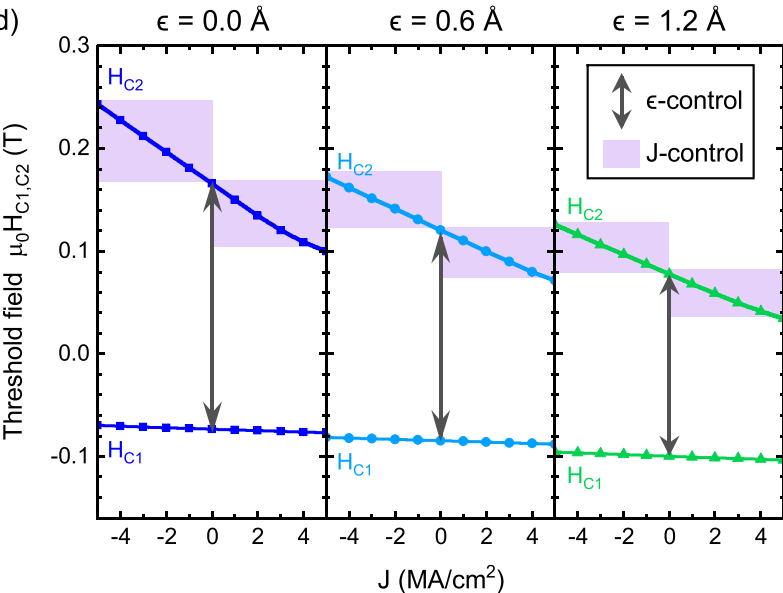

FIG. 4. Top: Angle dependence of DFT+JUNPY calculated current-driven spin-torque fields, $H_{\text {damp }}^{(J)} \sin \theta$, with writing current densities of $J= \pm 1.8 \mathrm{MA} / \mathrm{cm}^{2}$ for (a) $\mathrm{Co} / \mathrm{BDA} / \mathrm{Co}$ and (b) $\mathrm{Co} / \mathrm{BDT} / \mathrm{Co} \mathrm{SMMJs}$ under various tensile strains $\epsilon$. Bottom: Current dependence of DFT+JUNPY+LLG calculated $\mu_{0} H_{\mathrm{C} 1}$ and $\mu_{0} H_{\mathrm{C} 2}$ for (c) Co/BDA/Co and (d) Co/BDT/Co SMMJs under various tensile strains at zero temperature. The black vertical arrows and purple shaded areas represent the strain control and current control of the threshold fields, $H_{\mathrm{C} 1}$ and $H_{\mathrm{C} 2}$, respectively. The $H_{\mathrm{C} 1}\left(H_{\mathrm{C} 2}\right)$ is the threshold field required for P-to-AP (AP-to-P) magnetization switching.

(a) $\mathrm{Co} / \mathrm{BDA} / \mathrm{Co}(\epsilon=0.0 \AA)$
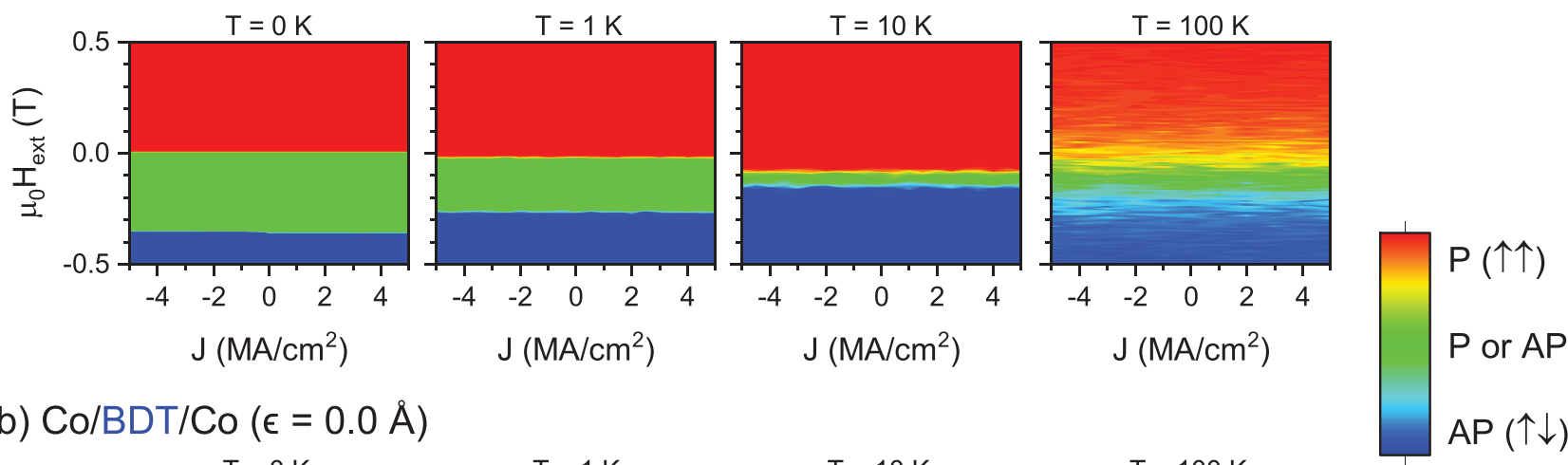

(b) $\mathrm{Co} / \mathrm{BDT} / \mathrm{Co}(\epsilon=0.0 \AA)$
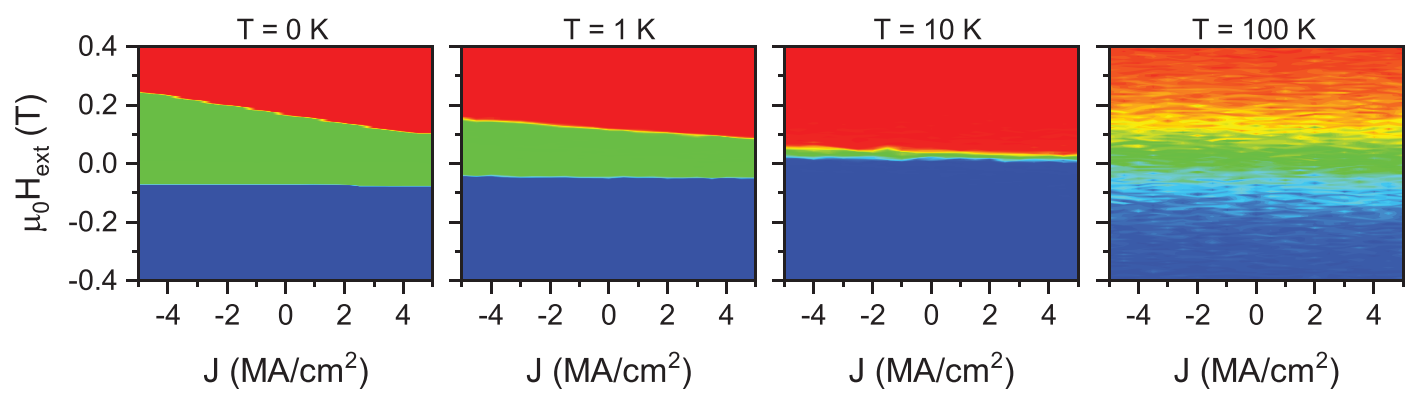

FIG. 5. Finite writing pulse phase diagrams for DFT+JUNPY+LLG calculated (a) Co/BDA/Co and (b) Co/BDT/Co SMMJs without strain at various temperatures ranging from 0 to $100 \mathrm{~K}$. The time step is $10 \mathrm{ps,} \mathrm{and} \mathrm{the} \mathrm{integration} \mathrm{time} \mathrm{is} 0.1 \mu \mathrm{s}$ in each field point. Each diagram is averaged with 10 identical simulations with different random noise. 
the hysteresis curves as a function of external magnetic field and applied current density are averaged with 10 identical calculations with different random noise. It is clear to find that the increase of the thermal effect gradually quenches the magnetization switching process when the temperature is below $100 \mathrm{~K}$. Comparing to the thermal stability of conventional oxide-based MTJs at room temperature [47], the free Co nanowire in SMMJs is too thin to retain its thermal stability. This is because the spin-torque effect mainly occurs at the interface and is proportional to the lateral area of the Co nanowire, while, the thermal field $\mathbf{H}_{\mathrm{th}}$ acting on the whole free Co nanowire. Therefore, our predicted spin-torque induced dual control of the EB effect and magnetic coercivity in SMMJs may be more applicable via the breaking junction techniques at low temperature, since experimental measurements of spin-polarized transport in molecular spintronics $[48,49]$ are mostly taken below $10 \mathrm{~K}$.

\section{CONCLUSION AND OUTLOOK}

It is worthwhile to mention that our predicted spin-torque fields and threshold fields may be sensitive to the break junction technique of real SMMJs, since there are some technical difficulties in controlling the contact geometry, hydrogen dissociation, and structural distortion during the stretching process. Our recent work [50] has employed the DFT calculation to comprehensively investigate the role of interfacial geometry and hydrogen dissociation in the spin transport properties of Co/BDA/Co SMMJs, either via (1) the covalent bonding between the $\mathrm{H}$-dissociated amine linker and spinpolarized Co apex atoms considered in this work, or by (2) the dative interaction between the $\mathrm{H}$-non-dissociated amine linker and Co apex atoms. The former covalent contact dominates the $\pi$ resonance interfacial spin selection that can be well preserved in $\mathrm{H}$-dissociated cases regardless of top, bridge, and hollow sites, as shown in Fig. 2 of Ref. [50]. This implies the existence of a strong FLST effect and EB effect, but may give very different angle dependence of the FLST and magnetization hysteresis curves, since the broadness of the spin-up transmission feature is strongly influenced by contact geometry. As for the issue of hydrogen dissociation with interfacial dative Co-N bonding, Fig. 3 of Ref. [50] reveals that the dative contact in the H-non-dissociated case not only destroys the spinterface coupling but also blocks the spin injection. Therefore, one can expect the significant reduction of the equilibrium FLST and EB effect, due to the suppression of the spin-up transmission feature. We believe that our multidisciplinary DFT+JUNPY+LLG calculation may provide important and practical implications to investigate the magnetic proximity and magnetization switching for novel molecular spintronics applications at low temperature.

In conclusion, we successfully combined the generalized LLG equation with the DFT+JUNPY calculated noncollinear spin-torque effects in the Co/BDA/Co and Co/BDT/Co SMMJs under a mechanical strain and an applied current density, which may provide a promising computational methodology to simulate the spin dynamics in real organic-based magnetic heterojunctions with strong ISF. The TB+JUNPY calculation is further employed to clarify the underlying mechanism of the crucial role of the linker and strain in the angle dependence of spin torques. We first predict that the significant EB effect in $\mathrm{Co} / \mathrm{BDA} / \mathrm{Co}$ SMMJs is purely dominated by the equilibrium FLST field, and the magnetic coercivity can only be controlled by tensile strain but not via applied current density of the order of $\mathrm{MA} / \mathrm{cm}^{2}$. As for the Co/BDT/Co SMMJs, the S linker provides a spin-down dominated ISF effect and slightly enhances both the equilibrium $H_{\perp}^{(0)}$ and current-driven $H_{\text {damp }}^{( \pm J)}$ of the same order. The interplay between them determines the magnetization switching and presents an important practical implication in the dual control of the EB effect and magnetic coercivity either by tensile strain or via applied current density of the order of $\mathrm{MA} / \mathrm{cm}^{2}$ at low temperature.

\section{ACKNOWLEDGMENTS}

We thank the National Center for High-performance Computing (NCHC) of the National Applied Research Laboratories (NARLabs) in Taiwan for providing computational and storage resources. This work is supported by the Ministry of Science and Technology (MOST 107-2633-M-008-004and 108-2628-M-008-004-MY3) and the National Center for Theoretical Sciences (NCTS).
[1] C. Leighton, M. R. Fitzsimmons, P. Yashar, A. Hoffmann, J. Nogués, J. Dura, C. F. Majkrzak, and I. K. Schuller, Two-Stage Magnetization Reversal in Exchange Biased Bilayers, Phys. Rev. Lett. 86, 4394 (2001).

[2] W.-G. Wang, M. Li, S. Hageman, and C. L. Chien, Electricfield-assisted switching in magnetic tunnel junctions, Nat. Mater. 11, 64 (2012).

[3] J. Nogués and I. K. Schuller, Exchange bias, J. Magn. Magn. Mater. 192, 203 (1999).

[4] B.-Y. Wang, C.-C. Chiu, W.-C. Lin, and M.-T. Lin, Enhanced perpendicular magnetic anisotropy in $\mathrm{Fe} / \mathrm{Mn}$ bilayers by incorporating ultrathin ferromagnetic underlayer through magnetic proximity effect, Appl. Phys. Lett. 103, 042407 (2013).

[5] P. K. Srivastava, Y. Hassan, H. Ahn, B. Kang, S.-G. Jung, Y. Gebredingle, M. Joe, M. S. Abbas, T. Park, J.-G. Park,
K.-J. Lee, and C. Lee, Exchange bias effect in ferro/antiferromagnetic van der Waals heterostructures, Nano Lett. 20, 3978 (2020).

[6] Y. Fan, K. J. Smith, G. Lüpke, A. T. Hanbicki, R. Goswami, C. H. Li, H. B. Zhao, and B. T. Jonker, Exchange bias of the interface spin system at the Fe/Mgo interface, Nat. Nanotechnol. 8, 438 (2013).

[7] P.-H. Lin, B.-Y. Yang, M.-H. Tsai, P.-C. Chen, K.-F. Huang, H.H. Lin, and C.-H. Lai, Manipulating exchange bias by spin-orbit torque, Nat. Mater. 18, 335 (2019).

[8] P. M. Haney, C. Heiliger, and M. D. Stiles, Bias dependence of magnetic exchange interactions: Application to interlayer exchange coupling in spin valves, Phys. Rev. B 79, 054405 (2009).

[9] Y.-H. Tang, N. Kioussis, A. Kalitsov, W. H. Butler, and R. Car, Controlling the Nonequilibrium Interlayer Exchange Coupling 
in Asymmetric Magnetic Tunnel Junctions, Phys. Rev. Lett. 103, 057206 (2009).

[10] C. Ortiz Pauyac, A. Kalitsov, A. Manchon, and M. Chshiev, Spin-transfer torque in spin filter tunnel junctions, Phys. Rev. B 90, 235417 (2014).

[11] Y.-H. Tang, F.-C. Chu, and N. Kioussis, Dual control of giant field-like spin torque in spin filter tunnel junctions, Sci. Rep. 5, 11341 (2015).

[12] Y.-H. Tang, Z.-W. Huang, and B.-H. Huang, Analytic expression for the giant fieldlike spin torque in spin-filter magnetic tunnel junctions, Phys. Rev. B 96, 064429 (2017).

[13] J. M. De Teresa, A. Barthélémy, A. Fert, J. P. Contour, F. Montaigne, and P. Seneor, Role of metal-oxide interface in determining the spin polarization of magnetic tunnel junctions, Science 286, 507 (1999).

[14] Z. H. Xiong, D. Wu, Z. V. Vardeny, and J. Shi, Giant magnetoresistance in organic spin-valves, Nature (London) 427, 821 (2004).

[15] J. C. Slonczewski, Currents, torques, and polarization factors in magnetic tunnel junctions, Phys. Rev. B 71, 024411 (2005).

[16] I. Theodonis, N. Kioussis, A. Kalitsov, M. Chshiev, and W. H. Butler, Anomalous Bias Dependence of Spin Torque in Magnetic Tunnel Junctions, Phys. Rev. Lett. 97, 237205 (2006).

[17] Y.-H. Tang, N. Kioussis, A. Kalitsov, W. H. Butler, and R. Car, Influence of asymmetry on bias behavior of spin torque, Phys. Rev. B 81, 054437 (2010).

[18] S. Sanvito, The rise of spinterface science, Nat. Phys. 6, 562 (2010).

[19] C.-H. Hsu, Y.-H. Chu, C.-I. Lu, P.-J. Hsu, S.-W. Chen, W.-J. Hsueh, C.-C. Kaun, and M.-T. Lin, Spin-polarized transport through single manganese phthalocyanine molecules on a Co nanoisland, J. Phys. Chem. C 119, 3374 (2015).

[20] M. Cinchetti, V. A. Dediu, and L. E. Hueso, Activating the molecular spinterface, Nat. Mater. 16, 507 (2017).

[21] X. Zhang, J. Tong, L. Ruan, X. Yao, L. Zhou, F. Tian, and G. Qin, Interface hybridization and spin filter effect in metalfree phthalocyanine spin valves, Phys. Chem. Chem. Phys. 22, 11663 (2020).

[22] R. Yamada, M. Noguchi, and H. Tada, Magnetoresistance of single molecular junctions measured by a mechanically controllable break junction method, Appl. Phys. Lett. 98, 053110 (2011).

[23] R. J. Brooke, C. Jin, D. S. Szumski, R. J. Nichols, B.-W. Mao, K. S. Thygesen, and W. Schwarzacher, Single-molecule electrochemical transistor utilizing a nickel-pyridyl spinterface, Nano Lett. 15, 275 (2015).

[24] S. Ding, Y. Tian, Y. Li, W. Mi, H. Dong, X. Zhang, W. Hu, and D. Zhu, Inverse magnetoresistance in polymer spin valves, ACS Appl. Mater. Interfaces 9, 15644 (2017).

[25] A. C. Aragonès, E. Medina, M. Ferrer-Huerta, N. Gimeno, M. Teixidó, J. L. Palma, N. Tao, J. M. Ugalde, E. Giralt, I. DíezPérez, and V. Mujica, Measuring the spin-polarization power of a single chiral molecule, Small 13, 1602519 (2017).

[26] G. Ke, C. Duan, F. Huang, and X. Guo, Electrical and spin switches in single-molecule junctions, InfoMat 2, 92 (2020).

[27] D. Liu, Y. Hu, H. Guo, and X. F. Han, Magnetic proximity effect at the molecular scale: First-principles calculations, Phys. Rev. B 78, 193307 (2008).
[28] S. Mandal and R. Pati, What determines the sign reversal of magnetoresistance in a molecular tunnel junction? ACS Nano 6, 3580 (2012).

[29] D. Li, R. Banerjee, S. Mondal, I. Maliyov, M. Romanova, Y. J. Dappe, and A. Smogunov, Symmetry aspects of spin filtering in molecular junctions: Hybridization and quantum interference effects, Phys. Rev. B 99, 115403 (2019).

[30] S. Li, Y. Wang, Y. Wang, S. Sanvito, and S. Hou, Highperformance spin filters based on 1,2,4,5-tetrahydroxybenzene molecules attached to bulk nickel electrodes, J. Phys. Chem. C 125, 6945 (2021).

[31] S. Haku, A. Ishikawa, A. Musha, H. Nakayama, T. Yamamoto, and K. Ando, Surface Rashba-Edelstein Spin-Orbit Torque Revealed by Molecular Self-Assembly, Phys. Rev. Appl. 13, 044069 (2020).

[32] Y.-H. Tang and B.-H. Huang, Manipulation of giant field-like spin torque in amine-ended single-molecule magnetic junctions, J. Phys. Chem. C 122, 20500 (2018).

[33] B. Q. Xu, X. L. Li, X. Y. Xiao, H. Sakaguchi, and N. J. Tao, Electromechanical and conductance switching properties of single oligothiophene molecules, Nano Lett. 5, 1491 (2005).

[34] M. Ratner, A brief history of molecular electronics, Nat. Nanotechnol. 8, 378 (2013).

[35] T. A. Su, M. Neupane, M. L. Steigerwald, L. Venkataraman, and C. Nuckolls, Chemical principles of single-molecule electronics, Nat. Rev. Mater. 1, 16002 (2016).

[36] P. Gehring, J. M. Thijssen, and H. S. J. van der Zant, Singlemolecule quantum-transport phenomena in break junctions, Nat. Rev. Phys. 1, 381 (2019).

[37] B.-H. Huang, C.-C. Chao, and Y.-H. Tang, Thickness dependence of spin torque effect in $\mathrm{Fe} / \mathrm{Mgo} / \mathrm{Fe}$ magnetic tunnel junction: Implementation of divide-and-conquer with firstprinciples calculation, AIP Adv. 11, 015036 (2021).

[38] The detailed information of our self-developed JUNPY package can be found at https://labstt.phy.ncu.edu.tw/junpy.

[39] P. Giannozzi, S. Baroni, N. Bonini, M. Calandra, R. Car, C. Cavazzoni, D. Ceresoli, G. L. Chiarotti, M. Cococcioni, I. Dabo, A. D. Corso, S. de Gironcoli, S. Fabris, G. Fratesi, R. Gebauer, U. Gerstmann, C. Gougoussis, A. Kokalj, M. Lazzeri, L. Martin-Samos et al., QUANTUM ESPRESSO: A modular and open-source software project for quantum simulations of materials, J. Phys.: Condens. Matter 21, 395502 (2009).

[40] J. P. Perdew, K. Burke, and M. Ernzerhof, Generalized Gradient Approximation Made Simple, Phys. Rev. Lett. 77, 3865 (1996).

[41] D. Waldron, L. Liu, and H. Guo, $A b$ initio simulation of magnetic tunnel junctions, Nanotechnology 18, 424026 (2007).

[42] J. Taylor, H. Guo, and J. Wang, Ab initio modeling of quantum transport properties of molecular electronic devices, Phys. Rev. B 63, 245407 (2001).

[43] Y. Ke, K. Xia, and H. Guo, Disorder Scattering in Magnetic Tunnel Junctions: Theory of Nonequilibrium Vertex Correction, Phys. Rev. Lett. 100, 166805 (2008).

[44] D. J. P. de Sousa, P. M. Haney, D. L. Zhang, J. P. Wang, and T. Low, Bidirectional switching assisted by interlayer exchange coupling in asymmetric magnetic tunnel junctions, Phys. Rev. B 101, 081404(R) (2020).

[45] J. Xiao, A. Zangwill, and M. D. Stiles, Macrospin models of spin transfer dynamics, Phys. Rev. B 72, 014446 (2005). 
[46] Y. H. Tang and C. J. Lin, Strain-enhanced spin injection in amine-ended single-molecule magnetic junctions, J. Phys. Chem. C 120, 692 (2016).

[47] A. A. Timopheev, R. Sousa, M. Chshiev, L. D. Buda-Prejbeanu, and B. Dieny, Respective influence of in-plane and out-of-plane spin-transfer torques in magnetization switching of perpendicular magnetic tunnel junctions, Phys. Rev. B 92, 104430 (2015).
[48] J. R. Petta, S. K. Slater, and D. C. Ralph, Spin-Dependent Transport in Molecular Tunnel Junctions, Phys. Rev. Lett. 93, 136601 (2004).

[49] L. Bogani and W. Wernsdorfer, Molecular spintronics using single-molecule magnets, Nat. Mater. 7, 179 (2008).

[50] K.-R. Chiang and Y.-H. Tang, Effect of contact geometry on spin transport in amine-ended single-molecule magnetic junctions, ACS Omega 6, 19386 (2021). 